\title{
Interacción entre el hongo endofítico Fusarium oxysporum cepa 162 y la bacteria endofítica Rhizobium etli cepa G12
}

\section{Interactions between the endophytic fungus Fusarium oxysporum strain 162 and the endophytic bacterium Rhizobium etli strain G12}

\begin{abstract}
Alfonso Martinuz
Representación en Nicaragua de la Organización de la Naciones Unidas para la Alimentación y la Agricultura (FAO), ORCID: https://orcid. org/0000-0002-3311-6646, Alfonso.MartinuzGuerrero@fao.org
\end{abstract}

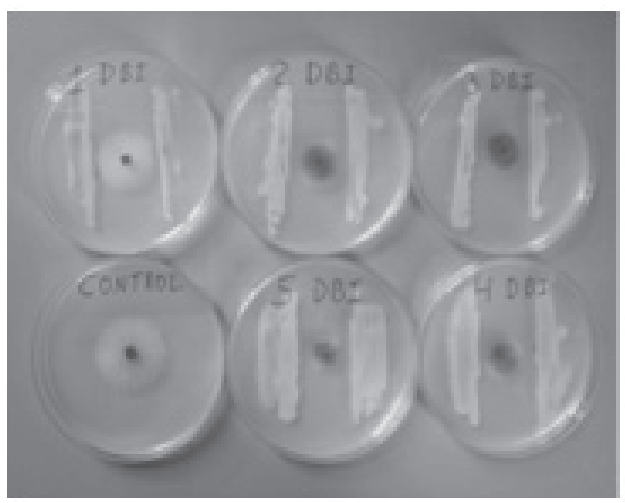

\section{RESUMEN}

Las cepas de Fusarium no patogénicas son importantes para la producción de cultivos, ya que algunos de ellos han mostrado un efecto de inducción de resistencia en las plantas colonizadas, predisponiendo el sistema inmune de las plantas a defenderse contra el ataque de plagas y enfermedades. Las bacterias endofíticas son aisladas de los tejidos internos de las plantas, siendo un grupo importante las rhizobacterias. Estudios han demostrado que la inoculación de estas bacterias en diferentes cultivos disminuyó la intensidad de enfermedades causadas por patógenos del suelo, resultando en la promoción de crecimiento y salud de las plantas. Sin embargo, la variabilidad en el nivel de control biológico de las plagas y enfermedades, asociadas a la aplicación simultanea de microorganismos endofíticos, es considerado como un factor limitante al usar agentes antagonistas para la protección de cultivos. En este contexto, la presente investigación evaluó la interacción entre los endofíticos Fusarium oxysporum Fo162 y Rizobium etli G12 al aplicarlos simultáneamente en experimentos in vitro e in vivo. Los resultados indicaron que la bacteria causó una reducción significativa en el crecimiento radial del hongo, así como en su capacidad de colonización radicular. Lo anterior, sugiere que es necesario evaluar la compatibilidad entre microorganismos benéficos, antes de utilizarlos en inoculaciones simultaneas como parte de una estrategia de control biológico.

Palabras clave: control biológico, resistencia inducida, inhibición, aplicaciones simultaneas, bioensayos.

\section{ABSTRACT}

Non-pathogenic Fusarium strains are important to crop production, since some of them have shown to induce resistance in colonized plants; thus, increasing the plant's ability to defend itself from pathogen and pest attack. Endophytic bacteria groups, as rhiozobacteria, are able to stimulate plant growth and resistance to pest and diseases also. Nevertheless, the inconsistency in the level of biocontrol of pest and diseases -often associated with simultaneous application of microorganisms to plants- is considered a limiting factor when using antagonistic fungi and bacteria for plant protection. Therefore, the present investigation evaluates the in vitro and in vivo interactions between the endophytic fungus Fusarium oxysporum strain Fo162 and the endophytic bacterium Rhizobium etli strain G12. The results indicate that the bacteria caused a significant reduction of the fungi radial growth, and thus its capacity of root colonization. This suggest that before using simultaneous inoculation of these beneficial microorganisms as a strategy of biological control, their compatibility should be evaluated.

Keywords: Biological control, induced resistance, inhibition, simultaneous treatments, bioassays.
Recibido: 16 de octubre del 2019 Aceptado: 24 de enero del 2020
Los artículos de la revista La Calera de la Universidad Nacional Agraria, Nicaragua, se comparten bajo términos de la licencia Creative Commons: Reconocimiento, No Comercial, Compartir Igual. Las autorizaciones adicionales a las aquí delimitadas se

(C) Copyright 2020. Universidad Nacional Agraria (UNA) - Organización de las Naciones Unidas para la Alimentación y la Agricultura (FAO) 


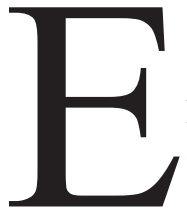

1 hongo Fusarium oxysporum es un habitante común y diverso del suelo. Este género de hongos incluye un número relevante de especies de hongos patógenos que afectan negativamente a cultivos de importancia económica a nivel mundial. Sin embargo, la mayoría de las cepas de $F$. oxysporum son saprófitos y normalmente son aislados de tejidos vegetales internos después de haber pasado por un proceso de desinfección superficial de las diferentes partes de las plantas (raíz, tallos y hojas). Las cepas de Fusarium no patogénicas son importantes en la producción de cultivos, ya que algunos de ellos han mostrado un efecto de inducción de resistencia en plantas colonizadas (Alabouvette, Schippers, Lemanceau, y Bakker, 1998; Larkin y Fravel, 1999; Pereira, Vieira, y Azevedo, 1999; Trouvelot, Olivain, Recorbet, Migheli, y Alabouvette, 2002; Sikora, Schaefer, y Dababat, 2007).

Las bacterias endofíticas son aisladas de los tejidos vegetales internos, a través de un proceso de desinfección superficial de diferentes partes de las plantas (raíz, tallo y hojas). No obstante, este tipo de bacterias tienen la habilidad de colonizar también la superficie de los tejidos vegetales (Hallman, Quadt-Hallmann, Miller, Sikora, y Lindow, 2001). El potencial de este tipo de microorganismos ha sido demostrado a través de varios estudios (Sturz, Christie, y Nowk, 2000; El-Batanony et al., 2007; Sikora et al., 2007), ya que inoculaciones de varios tipos de cepas de Rhizobacterias en diferentes cultivos resultó en la disminución de la agresividad de varias enfermedades causadas por patógenos del suelo.

La variabilidad en el nivel de control biológico de las plagas y enfermedades, asociadas a la aplicación de microorganismos endofíticos, es considerado como un factor limitante al usar agentes antagonistas para la protección de cultivos. La variabilidad en el nivel de control, se debe probablemente a que este tipo de estrategias se basan normalmente en la aplicación de un solo tipo de microorganismo. En estos casos, el control se limita a un período corto de tiempo como resultado de fluctuaciones bióticas y abióticas en el suelo (Dunne et al., 1998; Reimann, Hauschild, Hildebrandt, y Sikora, 2008). En consecuencia, el diseño de estrategias que involucren la aplicación simultanea de microorganismos benéficos han sido sugeridas como una medida para mejorar el nivel de control biológico (Dunne et al., 1998; El-Tarabily et al., 2000; Chaves, Pocasangre, Elango, Rosales, y Sikora, 2009).

Las asociaciones de diferentes tipos de microorganismos benéficos probablemente provean distintos mecanismos o combinación de mecanismos conducentes al control de diferentes plagas y enfermedades. Por ejemplo, inhibición de patógenos vía antibiosis, producción de metabolitos tipo sideróforos, competencia por nutrientes y/o espacio, inducción de resistencia, degradación o inhibición de la germinación del patógeno, reducción de factores de patogenicidad y promoción de crecimiento de las plantas por medio de la producción de fitohormonas (Deshwal, Dubey, y Maheshwari, 2003; El-Mehalawy, 2004; Sikora et al., 2007).

La interacción directa o indirecta de aplicaciones simultaneas de endofíticos, debe analizarse al implementar este tipo de estrategias de control, ya que aplicaciones de Tricoderma harzianum y micorrizas arbusculares resultó en inhibición del desarrollo y colonización radicular de las micorrizas (Wyss, Boller, y Wiemken, 1992). En otro experimento la colonización de la micorriza se vio favorecida en presencia de otra cepa de T. harzianum (Filion, St-Arnaud, y Fortin, 1999). Estudios adicionales, demostraron que al realizar aplicaciones combinadas de Glomus intraradices y Rhizobium etli G12 para el control de Meloidogyne incognita resultó en un efecto de control aditivo (Reimann et al., 2008); igualmente, aplicaciones simultaneas de F. oxysporum y Bacillus firmus causaron una reducción significativa de la cantidad de Radopholus similis que lograron penetrar las raíces de banano (Mendoza y Sikora, 2009). No obstante, la inoculación simultanea de los endofíticos $F$. oxysporum y Paecilomyces lilacinus no resultó en un nivel de control aditivo del nematodo $R$. similis en el cultivo de banano (Mendoza y Sikora, 2009). La combinación de F. oxysporum Fo162 y R. etli G12 mostró niveles similares de control (en comparación a aplicaciones individuales) de $M$. incognita y Aphis gossypii en tomate (Solanum lycopersicum L.) y zucchini (Cucurbita pepo L.) (Martinuz y Sikora, 2010).

Lo anterior parece indicar que la competencia entre microorganismos antagonistas, tanto dentro como sobre las raíces, conduce a la exclusión competitiva y por lo tanto a la reducción de la colonización y/o actividad de uno o más de los microorganismos que están siendo usados como agentes de control biológico. En este contexto, el objetivo de esta investigación fue evaluar la interacción entre los microorganismos endofíticos $F$. oxysporum Fo162 y $R$. etli G12 al aplicarlos simultáneamente en experimentos in vitro e in vivo.

\section{MATERIALES Y MÉTODOS}

Plantas. Para todos los experimentos se utilizaron plantas de tomate (cv. Moneymaker). Las semillas fueron esterilizadas sumergiéndolas durante un minuto en una solución de etanol al 75\% y luego por tres minutos en una solución de hipoclorito de sodio $(\mathrm{NaOCl})$. Luego las semillas fueron enjuagadas con agua esterilizada. Las semillas estériles fueron sembradas en bandejas múltiples (35 plantas) conteniendo arena esterilizada con autoclave a $121^{\circ} \mathrm{C}$ por una hora y aireadas durante dos días antes de su uso. Las bandejas de siembra se mantuvieron en una cámara de crecimiento a $25 \pm 3{ }^{\circ} \mathrm{C}, 16 \mathrm{~h}$ de luz día ${ }^{-1}$, $60-70 \%$ de humedad y fertilizadas semanalmente con cinco $\mathrm{ml}$ de un fertilizante comercial (14-10-14, $2 \mathrm{~g} \mathrm{l}^{-1}$ ). Después de 
cuatro semanas, las plántulas fueron trasplantadas a maseteros plásticos de $11 \mathrm{~cm}$ conteniendo $300 \mathrm{~g}$ de substrato esterilizado (mezcla de arena-suelo en proporción dos:uno vol:vol) y transferidas a un invernadero a $27 \pm 3^{\circ} \mathrm{C}$ y $16 \mathrm{~h}$ de luz día $\mathrm{a}^{-1}$.

F. oxysporum cepa Fo162. El hongo endofítico $F$. oxysporum cepa Fo162, subsecuentemente referido como Fo162, fue aislado originalmente de los tejidos radiculares de plantas de tomate cultivar Moneymaker en Kenia y conservado a $-80^{\circ} \mathrm{C}$, usando el sistema de preservación de esporas de microorganismos Cryobank, Mast Group Ltd., Merseyside, UK. El inóculo fue producido transfiriendo un pellet congelado, del sistema antes referido, a platos Petri ( 90 $\mathrm{mm}$ de diámetro) con agar de papa dextrosa (PDA; Difco, Sparks, MD) suplementados con $150 \mathrm{mg} \mathrm{l}^{-1}$ de sulfato de estreptomicina y cloranfenicol para evitar el crecimiento de bacterias. Luego el cultivo de Fo162 fue incubado por tres semanas a $25^{\circ} \mathrm{C}$ en la oscuridad. Los micelios y conidios fueron raspados de la superficie del agar con una espátula y suspendidos en agua esterilizada (autoclave a $121^{\circ} \mathrm{C}$ por 20 min). Las esporas fueron separadas del micelio colando la suspensión a través de cuatro capas de papel filtro fino. La concentración de las esporas fue determinada a través de un hemocitómetro (Thomas Scientific, Philadelphia, PA) y fue ajustada con agua estéril a $1 \times 10^{6}$ esporas $\mathrm{g}^{-1}$ de suelo.

R. etli cepa G12. La bacteria endofítica $R$. etli cepa G12, subsecuentemente referida como G12, fue aislada originalmente de la rizósfera de plantas de papa e inicialmente identificada como Agrobacterium radiobacter, pero reclasificada posteriormente como $R$. etli (HaskyGünther, Hoffmann-Hergarten, y Sikora, 1998). La bacteria fue conservada a $-80^{\circ} \mathrm{C}$ usando el sistema de preservación de esporas de microorganismos Cryobank, Mast Group Ltd., Merseyside, UK. Para la producción del inóculo, un pellet congelado conteniendo G12 fue cultivado en platos Petri (90 $\mathrm{mm}$ de diámetro) conteniendo agar solido King $\mathrm{B}$ durante $36 \mathrm{~h}$ a $28^{\circ} \mathrm{C}$ en la oscuridad (King, Warth, y Raney, 1954). Con este cultivo de bacterias se inoculó un Erlenmeyer conteniendo $100 \mathrm{ml}$ de medio liquido de King B y cultivado durante $36 \mathrm{~h}$ a $28^{\circ} \mathrm{C}$ en un agitador a $100 \mathrm{rpm}$ en la oscuridad. Luego la suspensión de bacterias fue centrifugada a 5000 $\mathrm{x}$ g por $20 \mathrm{~min}$ a $10^{\circ} \mathrm{C}$. Los pellets resultantes fueron suspendidos nuevamente en una solución de Ringer al 0.25 (Merck, Darmstadt, Germany) y la densidad óptica de las bacterias fue ajustada a dos a una longitud de onda de 560 $\mathrm{nm}$, representando aproximadamente $1.2 \times 10^{10}$ unidades formadoras de colonia (UFC) $\mathrm{ml}^{-1}$ (Hasky-Günther et al., 1998; Reitz et al., 2000; Reimann et al., 2008).

Evaluación de la interacción in vitro entre Fo162 y G12. Para evaluar la interacción in vitro entre los microorganismos endofíticos se realizaron dos experimentos.
Experimento 1. Interacción en diferentes medios de cultivo. Para evaluar la interacción entre Fo162 y G12 se utilizó un sistema de cultivo dual en platos Petri de nueve mm de diámetro conteniendo diferentes tipos de medios de cultivos. Para ello, cada plato Petri fue llenado con $25 \mathrm{ml}$ de uno de los siguientes medios de cultivo: i) Agar de Papa Dextrosa(APD), ii) Agar Sabouraud Dextrosa (ASD), iii) Agar de Soya Triptona (AST) y iv) Agar de Tejido de Raíces (ATR). Los medios APD, ASD y AST fueron preparados según las instrucciones del fabricante. Para preparar el medio ATR se utilizó $30 \mathrm{~g}$ de tejidos de raíces frescas de plantas de tomate de cuatro semanas de edad. Las raíces fueron maceradas y mezcladas con $500 \mathrm{ml}$ de agua destilada conteniendo $18 \mathrm{~g}$ de agar. La mezcla fue calibrada a un litro y esterilizada en la autoclave a $121^{\circ} \mathrm{C}$ por $20 \mathrm{~min}$. El bioensayo fue desarrollado de forma separada en cada uno de los medios de cultivo antes descritos. Para el experimento la bacteria fue colocada sobre el medio de cultivo en dos barras paralelas en cada plato Petri. Cada barra fue colocada a dos $\mathrm{cm}$ del centro del plato Petri y el control no fue inoculado con la bacteria (Figura 1). Posteriormente, la bacteria fue incubada por tres días (d) a $28^{\circ} \mathrm{C}$ en la oscuridad, inmediatamente después una sección de cinco mm de diámetro de APD con el hongo fue colocada en el centro de cada plato Petri (Figura 1) e incubado por un período de tres a cinco d a $28^{\circ} \mathrm{C}$ en la oscuridad. Cada tratamiento tuvo 10 repeticiones y el bioensayo fue replicado dos veces. El efecto de la bacteria sobre el desarrollo del hongo fue evaluado midiendo el crecimiento radial del micelio del hongo.
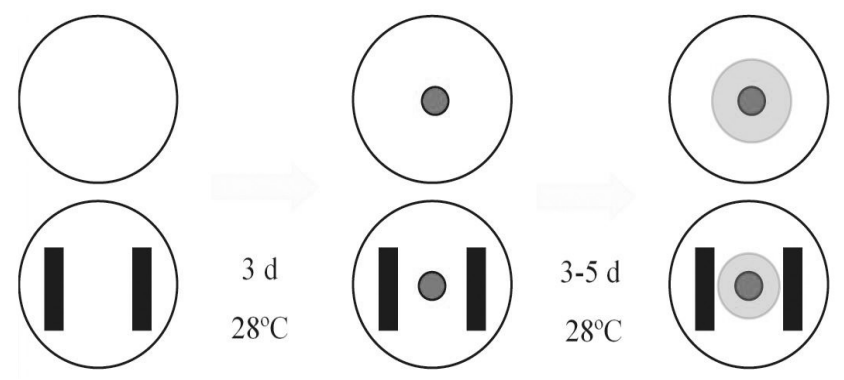

Figura 1. Diagrama de flujo usado para evaluar la interacción in vitro de G12 en el crecimiento de Fo162. Modificado de Reimann (2005).

Experimento 2. Efecto del tiempo de incubación bacteriano. La cantidad de compuestos antimicrobianos que producen las bacterias en medios de cultivo, se ve influenciada por el tiempo de fermentación o crecimiento bacteriano en dicho medio de cultivo. Para evaluar este factor se utilizó un sistema de cultivo dual en platos Petri de nueve $\mathrm{mm}$ de diámetro conteniendo $25 \mathrm{ml}$ de APD, la bacteria fue colocada sobre el medio de cultivo en dos barras paralelas en cada plato Petri. Cada barra fue colocada a dos $\mathrm{cm}$ del centro del plato 
Petri y el control no fue inoculado con la bacteria (Figura 2). Luego, los platos Petri fueron incubados por uno, dos, tres, cuatro y cinco d a $28^{\circ} \mathrm{C}$ en la oscuridad. Posteriormente, una sección de cinco mm de diámetro de APD con el hongo fue colocada en el centro de cada plato Petri e incubado por tres d a $28^{\circ} \mathrm{C}$ en la oscuridad (Figura 2). Cada tratamiento constó de seis repeticiones y el bioensayo fue replicado dos veces. Para evaluar el efecto del tiempo de incubación de la bacteria sobre el desarrollo del hongo se midió el crecimiento radial del hongo a los tres d de su exposición a la bacteria.

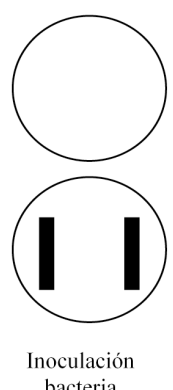

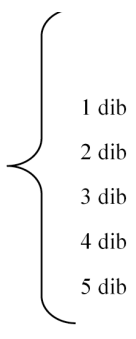

Periodo de incubación

dib $=$ Días de incubación bacteriana

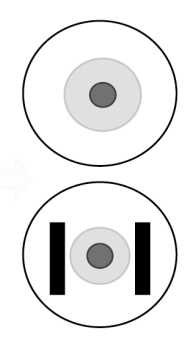

Crecimiento radial del hongo
Figura 2. Diagrama de flujo usado para evaluar el período de incubación de G12 en el desarrollo de Fo162. Modificado de Reimann (2005).

Evaluación de la interacción in vivo entre Fo162 y G12. Para evaluar la interacción in vivo entre los microorganismos endofíticos se desarrollaron dos experimentos.

Experimento 1. Inoculación individual de los antagonistas. Para evaluar la interacción in vivo entre Fo162 y G12 se llevó a cabo un bioensayo en el invernadero, en el cual las plantas recibieron inoculaciones del hongo y la bacteria de forma individual o combinadas. Para este experimento, plantas de tomate producidas según se describe arriba, fueron inoculadas de la siguiente manera: i) Fo162, ii) G12, iii) Fo162+G12 o iv) agua (como control). El Fo162 fue inoculado (con una pipeta) en tres orificios alrededor de la planta seleccionada con una suspensión de esporas (concentración $1 \times 10^{6} \mathrm{~g}^{-1}$ de suelo). La G12 fue aplicada alrededor de la planta seleccionada con cinco $\mathrm{ml} \mathrm{macetera}{ }^{-1}$ de una suspensión de bacterias (concentración $1.2 \times 10^{10} \mathrm{UFC} \mathrm{m}^{-1}$ ). Para el ensayo se utilizó un diseño completamente aleatorizado en un invernadero a $25 \pm 3^{\circ} \mathrm{C}$ con $16 \mathrm{~h}$ de luz artificial d $\mathrm{d}^{-1}$. Cada tratamiento consistió de seis repeticiones y el experimento fue desarrollado dos veces. La interacción entre los dos endofíticos fue evaluada a través de la medición del nivel de colonización de Fo162. La colonización de Fo162 fue determinada cuadro semanas después de su inoculación a través del método de la esterilización superficial de las raíces. Para ello, el sistema radicular de cada planta fue esterilizado sumergiéndolo en una solución de $\mathrm{NaOCl}$ al $1.5 \%$ por tres min, seguido de tres enjuagues en agua destilada esterilizada. Luego las raíces fueron pasadas sobre platos Petri con APD para verificar el proceso de esterilización (Schulz, Rommert, Dammann, Aust, y Strack, 1999). Las raíces esterilizadas fueron cortadas en secciones de $0.5 \mathrm{~cm}$ y 18 segmentos por cada tratamiento fueron seleccionados al azar y colocados en dos platos Petri (150 mm de diámetro) conteniendo APD. Después de cuatro a siete semanas de incubación a $25^{\circ} \mathrm{C}$ en la oscuridad, el crecimiento del hongo de cada sección de raíz fue identificado fenotípicamente como Fo162. Estos datos fueron usados para calcular el porcentaje de colonización del hongo [(número de secciones de raíces con crecimiento de Fo162/total de secciones de raíces colocadas en los dos platos Petri tratamiento $\left.\left.{ }^{-1}\right) * 100\right]$.

Experimento 2. Evaluación de la actividad sistémica a través del método de la raíz dividida. $L a$ influencia sistémica de G12 sobre la colonización radicular de Fo162 fue investigada por medio del método de la raíz dividida (Figura 3). Para el experimento se trasplantaron plántulas de tomate en maceteras conteniendo $300 \mathrm{~g}$ de substrato esterilizado (mezcla de arena:suelo en proporción dos:uno vol:vol) y mantenidas en un invernadero a $27 \pm 3^{\circ} \mathrm{C}$ con $16 \mathrm{~h}$ de luz artificial $\mathrm{d}^{-1}$. Dos semanas después, las plantas fueron separadas del sistema radicular (corte a $0.5 \mathrm{~cm}$ de la superficie del suelo) y el tallo fue cortado longitudinalmente cinco $\mathrm{cm}$. Luego, dos maceteras con $300 \mathrm{~g}$ del sustrato antes descrito, fueron colocadas una al lado de la otra (Figura 3) y cada mitad del tallo cortado anteriormente fue introducido en una de las meceteras antes indicadas (Figura 3). Las plantas se mantuvieron en el invernadero (bajo las condiciones indicadas previamente) por dos semanas. En este período de tiempo los tallos desarrollaron un sistema de raíces adventicios robusto y adecuado para llevar a cabo el ensayo. Cada macetera del modelo de raíz dividida fue etiquetada como "Inductor" y "Respuesta" (Figura 3). Cada "Inductor" fue inoculado con Fo162, G12 o agua (estos constituyen los tratamientos del experimento) a como se detalló en los acápites previos. Una semana después, cada "Respuesta" fue inoculada únicamente con Fo162. Las unidades de raíz dividida fueron organizadas según un diseño completamente aleatorizado en el invernadero bajo las condiciones antes detalladas. Cada tratamiento consistió de seis repeticiones y el ensayo fue desarrollado dos veces. Cuatro semanas después de inocular el "Inductor" el porcentaje de colonización de Fo162 en el lado "Respuesta" fue determinado a través del método de la esterilización superficial de las raíces descrito para el Experimento 1 de este acápite.

Análisis estadístico. Los datos de todos los experimentos fueron evaluados para comprobar los supuestos de 
normalidad y homogeneidad de varianza y luego se aplicó el análisis de varianza para un factor (ANOVA). Al encontrar diferencias significativas (prueba F) las medias por tratamiento fueron comparadas a través de la prueba de diferencias mínimas de Fisher $(p \leq 0.05)$. Los experimentos que consistían únicamente de dos tratamientos fueron analizados por medio de la prueba de t para comparación de dos muestras independientes $(p \leq 0.05)$. Para analizar el efecto in vitro de G12 sobre el crecimiento radial de Fo162 según el tiempo de incubación de la bacteria, se realizó a través de un análisis de regresión no lineal de la siguiente forma matemática: $\mathrm{Y}(\mathrm{t})=\alpha+(3 \cdot 6-\alpha) \exp \left(-\beta^{*} \mathrm{t}\right)$. Todos los análisis estadísticos fueron ejecutados usando el software InfoStat/Profesional versión 2009 (InfoStat Group, FCA., AR).

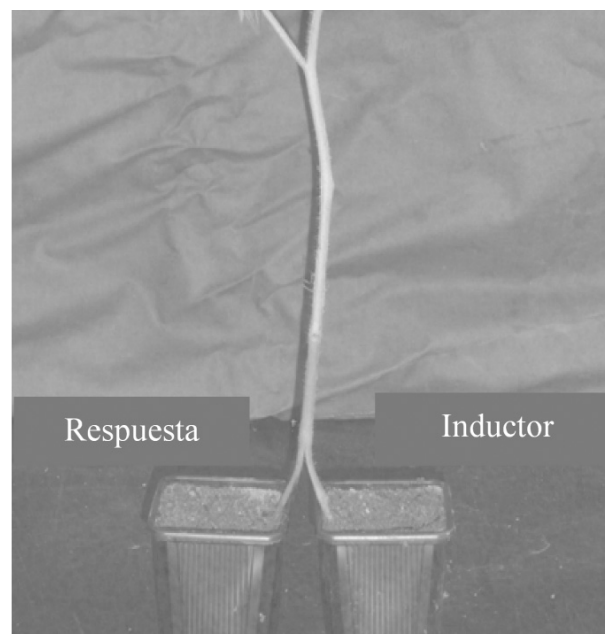

Figura 3. Método de la raíz dividida para evaluar la interacción sistémica de G12 sobre Fo162.
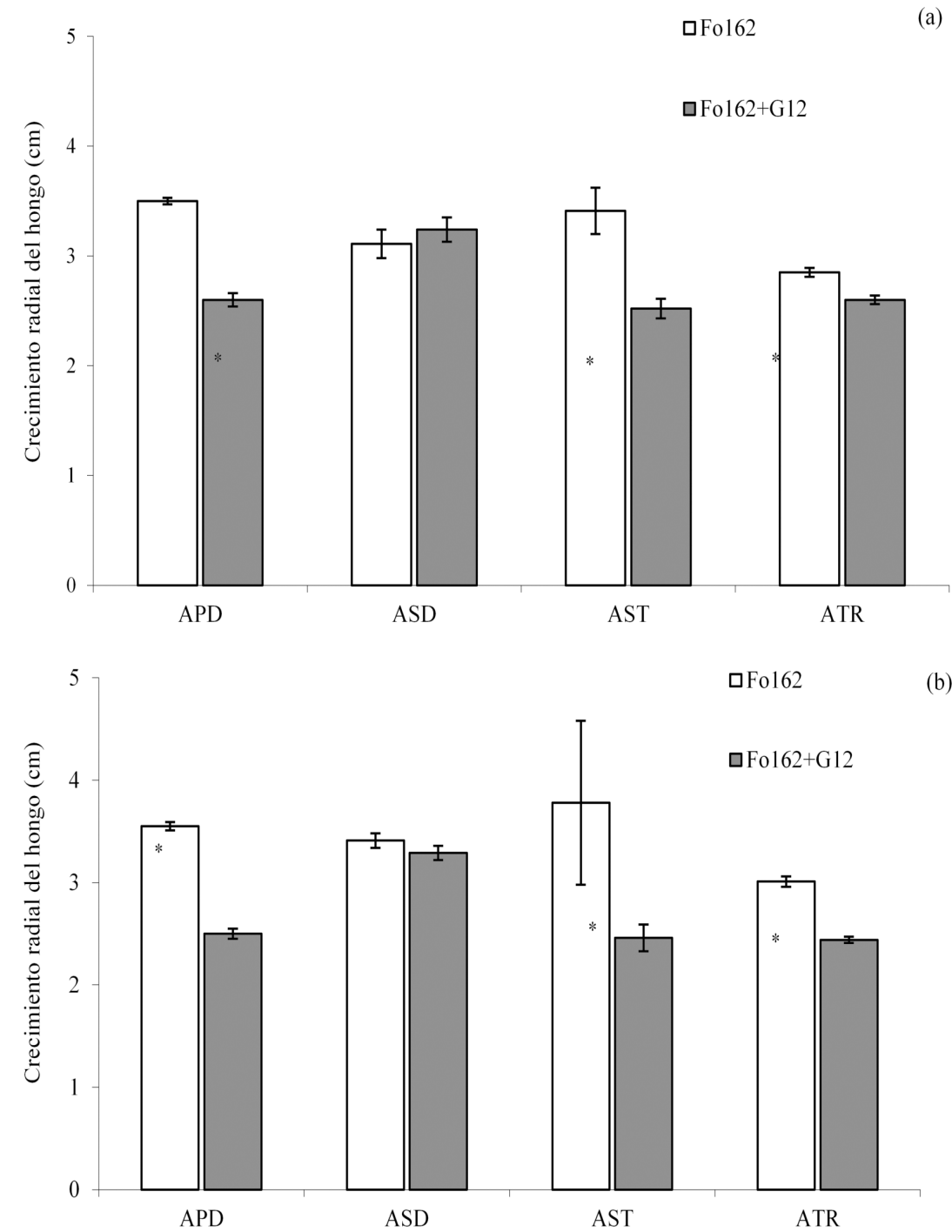

Figura 4. Efecto de G12 sobre el crecimiento radial de Fo162 en condiciones in vitro. Barras verticales representan el error estándar de la media. El asterisco indica diferencia significativa según la prueba de t para muestras independientes $(p \leq 0.05, \mathrm{n}=10)$. (a) Bioensayo 1 y (b) Bioensayo 2.

\section{RESULTADOS Y DISCUSIÓN}

Interacción en diferentes medios de cultivo. En el bioensayo del sistema de cultivo dual, se encontró que el crecimiento radial de Fo162 fue inhibido significativamente cuando el hongo se encuentra en presencia de G12, en comparación al testigo (Figura 4 a y b). Los resultados fueron similares en todos los medios de cultivo evaluados, excepto en el caso del ASD que no mostró diferencias significativas entre los tratamientos. Observaciones bajo el microscopio indicó que no había cambios morfológicos en el micelio de Fo162 al estar en presencia de G12.
Efecto del tiempo de incubación bacteriano. En este bioensayo se observó que el crecimiento radial de Fo162 decrece significativamente a medida que el tiempo de incubación bacteriano se incrementa (relación inversamente proporcional). Lo anterior fue demostrado a través de un análisis de regresión no lineal, siendo una relación negativa (Figura 5 a y b). En el primer Bioensayo (Figura 5a) el crecimiento radial del hongo decreció: 10, 17, 22, 26 y $28 \%$ $\mathrm{y}$ en el segundo Bioensayo (Figura 5b) se redujo: 22, 35, 43, 48 y $50 \%$ en comparación al testigo, cuando el tiempo de incubación bacteriano fue uno, dos, tres, cuatro o cinco días respectivamente. 


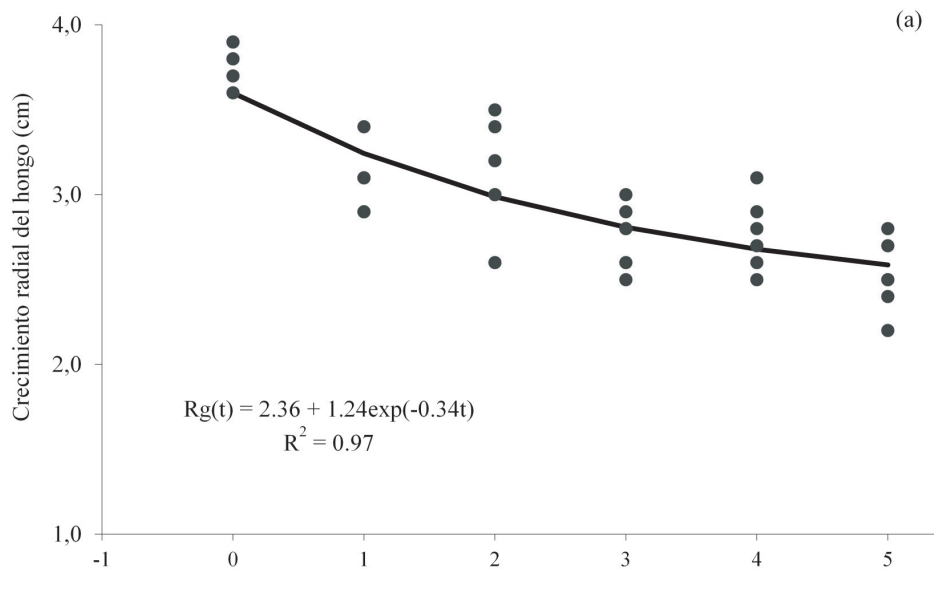

Días de incubación bacteriana

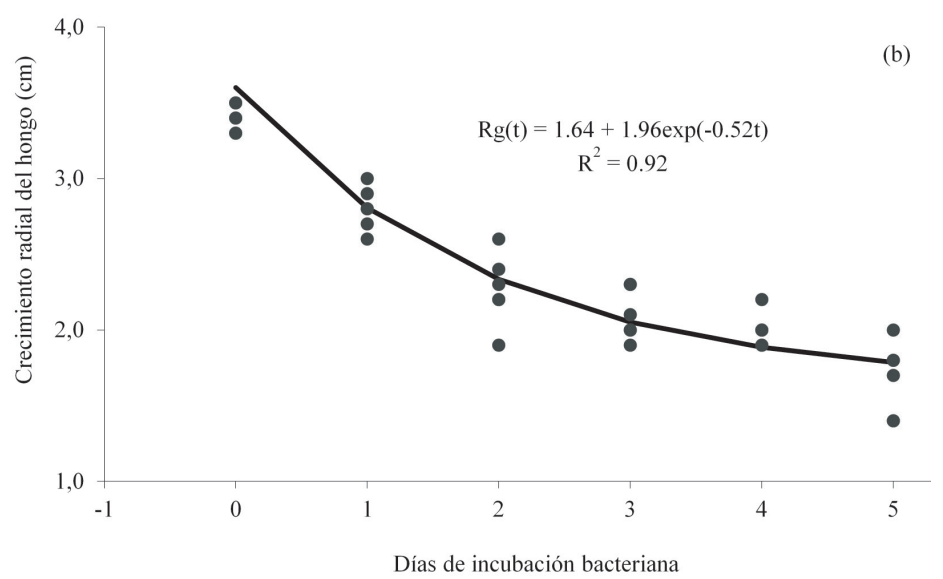

Evaluación de la actividad sistémica a través del método de raíz dividida. Cuatro semanas después de la inoculación del lado "Respuesta" del sistema de raíz dividida, se observó un efecto de inhibición sistémica de la colonización radicular de Fo162. En presencia de G12 en el lado "Inductor" del sistema, el porcentaje de colonización de Fo162 (lado "Respuesta") disminuyó en $50 \%$ en comparación al testigo en ambos experimentos (Cuadro 2). La inoculación de la sección "Inductor" del sistema ya sea con agua o con Fo162, no mostró diferencias significativas en la colonización del hongo en la sección "Respuesta" del sistema. En este caso el porcentaje de colonización de Fo162 fue de 18 y 22\% en los Experimentos 1 y 2 respectivamente. Lo anterior, resultó ser similar a los niveles de colonización obtenidos cuando el "Inductor" fue inoculado con agua, siendo 17 y $25 \%$ en ambos experimentos respectivamente (Cuadro 2).

Cuadro 2. Actividad sistémica de G12 en la colonización radicular de Fo162

\begin{tabular}{|c|c|c|}
\hline \multirow[b]{2}{*}{ Tratamiento } & Experimento 1 & Experimento 2 \\
\hline & \multicolumn{2}{|c|}{$\begin{array}{c}\text { colonización radicular (lado respuesta) } \\
(\%)\end{array}$} \\
\hline Fol62 & $18 \pm 2 \mathrm{a}$ & $22 \pm 5 \mathrm{a}$ \\
\hline G12 & $9 \pm 1 \mathrm{~b}$ & $10 \pm 2 b$ \\
\hline Agua & $16 \pm 2 \mathrm{a}$ & $25 \pm 4 \mathrm{a}$ \\
\hline
\end{tabular}

Datos representan la media \pm error estándar de la media. Medias con letras diferentes representan diferencias significativas según la prueba de diferencias mínimas de Fisher $(p \leq 0.05$, $\mathrm{n}=6)$.

Resultados de estudios previos relacionados a esta investigación (Martinuz, Schouten, Menjivar, y Sikora, 2012; Martinuz, Schouten, y Sikora, 2012; Martinuz, Schouten, y Sikora, 2013), indicaron que aplicaciones individuales y combinadas de Fo162 y G12 inducen la resistencia de las plantas a $M$. incognita en el sistema radicular y a Aphis gossypii en las hojas. Sin embargo, las aplicaciones combinadas de los microorganismos endofíticos no mostró niveles de control aditivos o sinérgicos del nematodo y el áfido. Existen evidencias de que la aplicación conjunta de dos antagonistas, con diferentes mecanismos de acción y nichos de colonización en la raíz, podría incrementar la eficacia de la supresión del nivel de infección de los nematodos (Reimann et al., 2008; Chaves et al., 2009). Por el contrario, algunos autores han reportado efectos de control de nematodos no aditivos al realizar aplicaciones combinadas de bacterias y hongos (Castillo et al., 2006; Mendoza y Sikora, 2009). A pesar de que el rol de microorganismos endofíticos específicos, especialmente hongos y bacterias, en el control de infecciones de insectos y nematodos han sido bien estudiados (Clay, 1989; Breen, 1994; Saikkonen, Faeth, Helander, y Sullivan, 1998; Azevedo, Maccheroni, Pereira,
Datos representan la media \pm error estándar de la media. El asterisco indica diferencia significativa entre los tratamientos de un mismo experimento, según la prueba de $\mathrm{t}$ para muestras independientes $(p \leq 0.05, \mathrm{n}=6)$. 
de Araújo, 2000; Sikora et al., 2007; Sikora, zum Felde, Mendoza, Menjivar, y Pocasangre, 2010), poco se sabe sobre el control biológico de plagas y enfermedades a través de la aplicación conjunta de diferentes tipos de microorganismos endofíticos (Gaylord, Preszler, y Boecklen, 1996; Diedhiou, Hallmann, Oerke, y Dehne, 2003; Martinuz y Sikora, 2010). Los experimentos in vitro e in vivo desarrollados en esta investigación fueron diseñados para analizar la interacción entre dos microorganismos benéficos que fueron aplicados al mismo tiempo en plantas de tomate.

Los experimentos de antibiosis desarrollados en esta investigación, resultaron en la reducción del crecimiento radial de Fo162 en presencia de G12. Esta reducción tuvo una correlación positiva con el tiempo de incubación de G12 (en un medio de cultivo sólido). Resultados similares fueron reportados por El-Botanony, Massoud, Mazen, y Monium (2007), quienes estudiaron in vitro e in vivo el efecto inhibitorio de filtrados de cultivos de Rhizobium spp. en el desarrollo de enfermedades del suelo, incluyendo $F$. oxysporum. Ellos encontraron que el efecto de inhibición, expresado como reducción del crecimiento del micelio del hongo patógeno, incrementa conforme aumenta la concentración de los filtrados de la bacteria. La conclusión del estudio fue que la bacteria endofítica analizada, ejerce un efecto antagonista sobre algunos hondos patógenos del suelo.

En ésta investigación, el efecto inhibitorio observado entre G12 sobre Fo162 podría estar relacionado, en parte, a la acumulación de metabolitos tóxicos producidos por la bacteria endofítica mientras se reproduce en los exudados de la planta. La falta de un nivel de control biológico sinérgico o aditivo, observado en esta investigación, podría estar relacionado al hecho de que ambos microorganismos fueron aplicados al mismo tiempo, causando competencia por espacio y nutrientes. Para evaluar esta hipótesis, se diseñó un experimento para analizar la influencia de G12 en la colonización radicular de Fo162. Los resultados de este experimento mostraron menor porcentaje de colonización de Fo162 cuando es aplicado en asociación con G12.

La forma en que estos microorganismos endofíticos colonizan las raíces, podría ser un tema de importancia en la interacción de ambos. Olivian et al., (2003) estudiaron la colonización de F. oxysporum cepa Fo47 en raíces de lino. Reportaron que el hongo colonizó activamente la superficie de las raíces. Posteriormente, el hongo penetró las células de la epidermis para colonizar abundantemente la hipodermis. Por otro lado, Hallmann et al. (2001) estudiaron la colonización interna y externa de las raíces de papa y Arabidopsis por parte de G12. El estudio se realizó a través de un plásmido promotor de trp que provoca una fluorescencia verde. Encontraron que la bacteria coloniza toda la superficie de las raíces, pero la colonización se concentra principalmente en las puntas de la raíz y las raíces laterales recién formadas. La colonización interna se concentró particularmente en las células de la epidermis, así como dentro o cerca del sistema vascular de la raíz. Además, Diedhiou et al. (2003) co-inocularon Glomus coronatum y Fo162 en un intento de incrementar el nivel de biocontrol del nematodo $M$. incognita. Los autores del estudio, encontraron que la presencia de Fo162 estimuló la colonización de $G$. coronatum y que las raíces ya colonizadas por G. coronatum, no pudieron ser colonizadas internamente por Fo162.

La resistencia sistémica inducida ha sido reportada como un mecanismo de control de G12 (Hasky-Günther et al., 1998). Este tipo de mecanismo puede afectar el desarrollo de hongos a como fue demostrado por Mwangi, Hauschild, Mutitu, y Sikora (2002). Por lo tanto, no se puede excluir la posibilidad de que este mecanismo sea un factor que afectó la interacción entre Fo162 y G12. Por esta razón, se diseñó el experimento de raíz dividida para analizar el efecto sistémico que causa G12 sobre la capacidad del Fo162 de colonizar el sistema radicular. Los resultados del experimento mostraron una reducción significativa en el porcentaje de colonización del Fo162, cuando el "Inductor" del sistema de raíz divida fue inoculado con G12. Resultados similares fueron reportados por Liu, Kloepper, y Tuzun (1995), quienes usaron un sistema de raíces divididas para estudiar la resistencia sistémica inducida por Pseudomonas putida y Serratia marcescens hacia Fusarium oxysporum f. sp. cucumerinum. Los autores encontraron, que ambas bacterias causaron una disminución en el desarrollo de la enfermedad, así como una reducción en la colonización de la raíz por parte del patógeno. No obstante, los autores no pudieron determinar si la resistencia sistémica inducida estaba relacionada a sustancias translocables inducidas o producidas por las bacterias.

Por otra parte, Reitz et al. (2000) usando experimentos de raíz dividida demostraron que lipopolisacáridos producidos por G12, funcionaron como un agente estimulador de la resistencia inducida contra Globodera pallida en plantas de papas (Solanum tuberosum L.). Además, van Peer y Schippers (1992) encontraron que lipopolisacáridos producidos por Pseudomonas spp no patogénicas fueron capaces de inducir resistencia sistémica hacia F. oxysporum f. sp. dianthi en plantas de clavel. Mwangi et al. (2002) reportaron resistencia sistémica inducida de Bacillus sphaericus B43 contra el patógeno F. oxysporum f.sp. lycopersici en tomate. Los autores encontraron que la actividad de la peroxidasa en los tallos de las plantas tratadas con la bacteria, incrementó significativamente en un período corto de tiempo, mientras que la actividad de la $\beta-1,3-$ glucanasa no fue afectada por la presencia de la bacteria. 


\section{CONCLUSIONES}

Los experimentos de antibiosis mostraron que la bacteria (G12) causó una reducción significativa en el crecimiento radial del hongo (Fo162). Esta reducción estuvo correlacionada positivamente con el período de incubación de la bacteria.

Los experimentos de invernadero en donde se evaluó aplicaciones simultaneas de Fo162 y G12, resultaron en la reducción del porcentaje de colonización radicular del Fo162, indicando que el hongo puede ser afectado directamente por la bacteria.
El experimento de raíz dividida indicó que las inoculaciones simultaneas, pero físicamente separadas, de Fo162 y G12 reducen la capacidad del hondo de colonizar las raíces, indicando que puede ser afectado de forma sistémica por la bacteria.

En general, los resultados sugirieron que es necesario llevar a cabo pruebas de compatibilidad entre microorganismos benéficos, antes de utilizarlos en inoculaciones simultaneas como parte de una estrategia de control biológico.

\section{REFERENCIAS BIBLIOGRÁFICAS}

Alabouvette, C., Schippers, B., Lemanceau, P., \& Bakker, P.A.H.M. (1998). Biological control of Fusarium Wilts: toward development of commercial products. In: G.J. Boland; L.D. Kuykendall (eds). Plant-microbe interaction and biological control. Marcel Dekker, New York, USA. pp 15-36.

Azevedo, J.L., Maccheroni, W., Pereira, J.O., \& de Araújo, W.L. (2000). Endophytic microorganisms: a review on insect control and recent advances on tropical plants. Electronic Journal of Biotechnology, 3, 40-65.

Breen, J.P. (1994). Acremonium endophytc interactions with enhanced resistance to insects. Annual Review of Entomology, $39,401-423$.

Castillo, P., Nico, I.A., Azcón-Aguilar, C., Del Río Rincón, C., Calvet, C., \& Jiménez-Díaz, R.M. (2006). Protection of olive planting stocks against parasitism of root-knot nematodes by arbuscular mycorrhizal fungi. Plant Pathology, 55, 705-713.

Chaves, N., Pocasangre, L.E., Elango, F., Rosales, F.E., \& Sikora, R.A. (2009). Combining endophytic fungi and bacteria for the biocontrol of Radopholus similis (Cobb) Thorne and for effects on plant growth. Scientia Horticulturae, 122, $472-478$.

Clay, K. (1989). Clavicipitaceous endophytes of grasses: their potential as biocontrol agents. Mycological Research, 92 , 1-12.

Deshwal, V.K., Dubey, R.C., \& Maheshwari, D.K. (2003). Isolation of plant growth-promoting strains of Bradyrhizobium (Arachis) sp. with biocontrol potential against Macrophomina phaseolina causing charcoal rot of peanut. Current Science, 84, 443-448.

Diedhiou, P.M., Hallmann, J., Oerke, E.C., \& Dehne, H.W. (2003). Effects of arbuscular mycorrhizal fungi and a non-pathogenic Fusarium oxysporum on Meloidogyne incognita infestation of tomato. Mycorrhiza, 13, 199-204.

Dunne, C., Moenne-Loccoz, Y., Mccarthy, J., Higgins, P., Powell, J., Dowling, D.N., \& O’gara, F. (1998). Combining proteolytic and phloroglucinol-producing bacteria for improved biocontrol of Pythium-mediated damping-off of sugar beet. Plant Pathology, 47, 299-307.

El-Batanony, N.H., Massoud, O.N., Mazen, M., \& El-Monium, A. (2007). The inhibitory effects of cultural filtratesof some wild Rhizobium spp. on some faba bean root rot pathogens and their antimicrobial synergistic effect when combined with Arbuscular mycorrhiza (Am). World Journal of Agricultural sciences, 3, 721-730.

El-Mehalawy, A.A. (2004). The Rhizosphere yeast fungi as biocontrol agents for wilt disease of kidney bean caused by Fusarium oxysporum. International Journal of Agriculture and Biology, 6, 310-316.

El-Tarabily, K.A., Soliman, M.H., Nassar, A.H., Al-Hassani, H.A., Sivasithamparam, K., Mckenna, F., \& Hardy, G. (2000). Biological control of Sclerotinia minor using a chitinolytic bacterium and actinomycetes. Plant Pathology, 49, 573-583.

Filion, M., St-Arnaud, M., \& Fortin, J.A. (1999). Direct interaction between the arbuscular mycorrhizal fungus Glomus intraradices and different rhizosphere microorganisms. New Phytologist, 141, 525-533.

Gaylord, E.S., Preszler, R.W., \& Boecklen, W.J. (1996). Interactions between host plants, endophytic fungi, and a phytophagous insect in an oak (Quercus grisea x Q. gambelii) hybrid zone. Oecologia, 3, 336-342.

Hallmann J., Quadt-Hallmann, A., Rodriguez-Kabana, R., \& Kloepper, J.W. (1998). Interactions between Meloidogyne incognita and endophytic bacteria in cotton and cucumber. Soil Biology and Biochemistry, 30, 925-937.

Hallmann, J., Quadt-Hallmann, A., Miller, W.G., Sikora, R.A., \& Lindow, S.E. (2001). Endophitic colonization of plants by the biocontrol agent Rhizobium etli G12 in relation to Meloidogyne incognita infection. Phytopathology, 91, 415-422.

Hasky-Günther, K., Hoffmann-Hergarten, S., \& Sikora, R.A. (1998). Resistance against the potato cyst nematode Globodera pallida systemically induced by the rhizobacteria Agrobacterium radiobacter (G12) and Bacillus sphaericus (B43). Fundamental and Applied Nematology, 21, 511-517.

King, E.O., Warth, M., \& Raney, E.D. (1954). Two simple media for the demonstration of procyanin and fluorescin. Journal of Laboratory and Clinical Medicine, 44, 301-307.

Larkin, R.P., \& Fravel, D.R. (1999). Mechanisms of action and dose-response relationships governing biological control of Fusarium wilt of tomato by non-pathogenic Fusarium spp. Phytopathology, 89, 1152-1161.

Liu, L., Kloepper J., \& Tuzun, S. (1995). Induction of systemic resistance in cucumber against Fusarium wilt by plant growth-promoting rhizobacteria. Phytopathology, 85, 695-698. 
Martinuz, A., \& Sikora, R.A. (2010). Biological control of root-knot nematodes and sucking insects by mutualistic endophytes in tomato and squash. Journal of Plant Diseases and Protection, 117, 137 [Abstract].

Martinuz, A., Schouten, A., \& Sikora, R.A. (2012). Effectiveness of systemic resistance toward Aphis gossypii (Hom., Aphididae) as induced by combined applications of the endophytes Fusarium oxysporum Fo162 and Rhizobium etli G12. Biological Control, 62, 206-212.

Martinuz, A., Schouten, A., Menjivar, R.D., \& Sikora, R.A. (2012). Systemically induced resistance and microbial competitive exclusion: Implications on biological control. Phytopathology, 102, 260-266.

Martinuz, A., Schouten, A., \& Sikora, R.A. (2013). Post-infection development of Meloidogyne incognita on tomato treated with the endophytes Fusarium oxysporum strain Fo162 and Rhizobium etli strain G12. BioControl, 58, 95-104.

Mendoza, A.R., \& Sikora, R.A. (2009). Biological control of Radopholus similis in banana by combined application of the mutualistic endophyte Fusarium oxysporum strain 162, the egg pathogen Paecilomyces lilacinus strain 251 and the antagonistic bacteria Bacillus firmus. BioControl, 54, 263-272.

Mwangi, M., Hauschild, R., Mutitu, E.W., \& Sikora, R.A. (2002). Rhizobacteria induced changes in tomato metabolism and their relationship with induced resistance against Fusarium oxysporum f.sp. lycopersici. Communications in agricultural and applied biological sciences, 67, 145-147.

Olivain C., Trouvelot, S., Binet, M.N., Cordier, C., Pugin, A., \& Alabouvette, C. (2003). Colonization of fax roots and early physiological responses of flax cells inoculated with pathogenic and nonpathogenic strains of Fusarium oxysporum. Applied and Environmental Microbiology, 69, 5453-5462.

Pereira, J.O., Vieira, M.L., \& Azevedo, J.L. (1999). Endophytic fungi from Musa acuminata and their reintroduction into axenic plants. World Journal of Microbiology \& Biotechnology, 15, 37-40.

Reimann, S. (2005). The interrelationships between rhizobacteria and arbuscular mycorrhizal fungi and their importance in the integrated management of nematodes and soilborne plant pathogens. Ph.D. thesis, University of Bonn, Germany.

Reimann, S., Hauschild, R., Hildebrandt, U., \& Sikora, R.A. (2008). Interrelationship between Rhizobium etli G12 and Glomus intraradices and multitrophic effects in the biological control of the root-knot nematode Meloidogyne incognita on tomato. Journal of Plant Diseases and Protection, 115, 108-113.

Reitz, M., Rudolph, K., Schroeder, I., Hoffmann-Hergarten, S., Hallmann, J., \& Sikora, R.A. (2000). Lipopolysaccharides of Rhizobium etli strain G12 act in potato roots as an inducing agent of systemic resistance to infection by the cyst nematode Globodera pallida. Applied and Environmental Microbiology, 66, 3515-3518.

Saikkonen, K., Faeth, S.H., Helander, M., \& Sullivan, T.J. (1998). Fungal endophytes: A continuum of interactions with host plants. Annual Review of Ecology and Systematics, 29, 319-343.

Schulz, B., Rommert, A.K. Dammann, U., Aust, H.J., \& Strack, D. (1999). The endophyte-host interaction: a balanced antagonism? Mycological Research, 10, 1275-1283.

Sikora, R.A., Schaefer, K., \& Dababat, A. (2007). Modes of action associated with microbially induced in planta suppression of plantparasitic nematodes. Australasian Plant Pathology, 36, 124-134.

Sikora, R.A., zum Felde, A., Mendoza, A., Menjivar, R., \& Pocasangre, L. (2010). In planta suppressiveness to nematodes and long term root health stability through biological enhancement - do we need a cocktail? Acta Horticulturae (ISHS), 879, 553-560.

Sturz, A.V., Christie, B.R., \& Nowk, J. (2000). Bacterial endophytes potential role in developing sustainable system of crop production plants. Sciences, 19, 1-30.

Trouvelot, S., Olivain, C., Recorbet, G., Migheli, Q., \& Alabouvette, C. (2002). Recovery of Fusarium oxysporum Fo47 mutants affected in their antagonistic activity after transposon mutagenesis. Phytopathology, 92, 936-945.

Van Peer, R., \& Schippers, B. (1992). Lipopolysaccharides of plant growth promoting Pseudomonas spp. strain WCS417r induce resistance in carnation to Fusarium wilt. Neth. Journal of Plant Pathology, 98, 129-139.

Wyss, P., Boller, T.H., \& Wiemken, A. (1992). Testing the effect of biological control agents on the formation of vesicular arbuscular mycorrhiza. Plant Soil, 147, 159-162. 